\title{
Statistical hadronization of supercooled quark-gluon plasma
}

\author{
A. Keränen* \\ Department of Physical Sciences, P.O. Box 3000, FIN-90014 Oulu, University of Oulu, Finland
}

L. P. Csernai ${ }^{\dagger}$

Section for Theoretical Physics, Department of Physics, University of Bergen, Allégt. 55, 5007 Bergen, Norway and KFKI Research Institute for Particle and Nuclear Physics, P.O. Box 49, 1525 Budapest, Hungary

V. Magas ${ }^{\dagger}$

Center for Physics of Fundamental Interactions (CFIF), Physics Department, Instituto Superior Tecnico, Avenida Rovisco Pais, 1049-001 Lisbon, Portugal

J. Manninen ${ }^{\S}$

Department of Physical Sciences, P.O. Box 3000, FIN-90014 Oulu, University of Oulu, Finland

(Received 6 May 2002; revised manuscript received 10 September 2002; published 24 March 2003)

\begin{abstract}
The fast simultaneous hadronization and chemical freeze-out of supercooled quark-gluon plasma, created in relativistic heavy ion collisions, can lead to the reheating of the expanding matter and to the change in a collective flow profile. We use the assumption of statistical nature of the hadronization process, and study quantitatively the freeze-out in the framework of hydrodynamical Bjorken model with different simple quarkgluon plasma equations of state.
\end{abstract}

DOI: 10.1103/PhysRevC.67.034905

\section{INTRODUCTION}

The hydrodynamical models have been used extensively to study the evolution of the hot, strongly interacting matter created in relativistic heavy ion collisions. These models apply on the space-time region, where the initial, hard processes have reached the stage where the local thermal equilibrium can be assumed, and the strong interactions between constituent particles are very frequent.

On the other hand, the thermal statistical models applied to describe the final hadron abundances have been very successful for nucleus-nucleus $(A-A)$ [1-5], proton-nucleus $(p-A)[6]$, and even for elementary $p-p, p-\bar{p}$, and $e^{+}-e^{-}$ $[7,8]$ reactions. The latter ones, particularly, suggest the statistical nature of the hadronization process.

In this work, we study the fast hadronization and chemical freeze-out (CFO) of locally thermalized quark-gluon plasma (QGP) described by hydrodynamical evolution. This process is idealized by sudden hadronization over a threedimensional hypersurface. The matter crossing the hypersurface is controlled by conservation of energy-momentum and relevant current conservation laws, and additionally, by assumption of apparent thermal and chemical equilibrium distribution of the resulting hadron gas ( $\mathrm{HG})$. By apparent equilibrium distribution we mean that although there is clearly no room for kinetic equilibration in elementary collisions - or in fast, simultaneous phase transition and CFO in nuclear collisions - the final hadron spectra are dictated by maximum entropy bound by conservation of energy and charge densities.

\footnotetext{
*Electronic address: antti.keranen@oulu.fi

†Electronic address: csernai@fi.uib.no

*Electronic address: vladimir@gtae3.ist.utl.pt

${ }^{\S}$ Electronic address: jaakko.manninen@oulu.fi
}

PACS number(s): 24.10.Pa, 24.10.Nz, 25.75.Dw, 25.75.Ld

It is shown that the first-order quasistatic phase transition is far too slow $[9,10]$ to give the hadron chemical decoupling time of $t_{\mathrm{CFO}} \lesssim 10 \mathrm{fm} / c$ determined experimentally, see Ref. [11] and references therein. Thus, in order to avoid the entropy decrease in the nonequilibrium hadronization, QGP must be allowed to supercool, i.e., to develop mechanical instability before the phase transition [11]. Although the simultaneous phase transition and $\mathrm{CFO}$ are assumed to be a nonequilibrium process, we can still exploit the apparent equilibrium parametrization-the consequence of statistical nature of hadronization.

The purpose of this work is more the qualitative understanding of the phenomenon than quantitative comparison with experiment. We use simplest, but not meaningless, geometry and equations of state (EoS). Nevertheless, our consideration leads to nontrivial results.

We will show that the shocklike hadronization of supercooled QGP leads to the change in collective flow profile and reheating of the system. We study the system with various choices of the EoS on both sides of the FO hypersurface.

\section{THE CHEMICAL FREEZE-OUT PROCESS}

The fast hadronization process, idealized to take place on the zero-volume hypersurface, leads to a discontinuity in energy-momentum and charge conservation equations. In general, this leads not only to change in density quantities in LRF, but to the change in flow velocity profile as well $[12,13]$. In order to have a sudden or rapid hadronization, the system should be out of equilibrium considerably. Then processes such as spinodal decomposition or surface instabilities, like viscous fingering [14], can result in rapid, nonthermal hadronization.

Denoting post-freeze-out quantities by subscript FO, the energy-momentum $(T)$ conservation at the surface element 
$d \sigma_{\nu}$ leads to a set of four equations,

$$
T^{\mu \nu} d \sigma_{\nu}=T_{\mathrm{FO}}^{\mu \nu} d \sigma_{\nu}
$$

We assume that the electric charge and the baryon charge are locally connected because the electromagnetic interaction has lesser importance in our study. Then we do not have to deal with the electric charge separately. Thus we are left with local baryon number and strangeness conservation equations:

$$
\begin{aligned}
& N_{B}^{\nu} d \sigma_{\nu}=\left(N_{B}^{\nu}\right)_{\mathrm{FO}} d \sigma_{\nu}, \\
& N_{S}^{\nu} d \sigma_{\nu}=\left(N_{S}^{\nu}\right)_{\mathrm{FO}} d \sigma_{\nu} .
\end{aligned}
$$

Here, we restrict our considerations to timelike FO hypersurfaces in order to avoid currents entering from post-FO to pre-FO side. The complications in the general case, where the spacelike FO is included, are discussed extensively in Refs. [12,13]. For the timelike hypersurface, there is always a proper Lorentz transformation for each point $x$ such that solving Eqs. (1)-(3) can be carried out in a frame, where flow velocities are $u(x)=u_{0}(1,0,0, v)$ and $u_{\mathrm{FO}}(x)$ $=\left(u_{0}\right)_{\mathrm{FO}}\left(1,0,0, v_{\mathrm{FO}}\right)$ on pre- and post-FO sides, respectively. Here we denote the Lorentz $\gamma$ factor by $u_{0}$. After solving the $\mathrm{FO}$ equations, the actual flow velocity $u_{\mathrm{FO}}=\left(u_{0}\right)_{\mathrm{FO}}\left(1, \vec{v}_{\mathrm{FO}}\right)$ is obtained by a simple spatial rotation. Provided with these tools, only two of the four equations in Eq. (1) are independent.

Strangeness is one of the first and basic QGP signals [15]. Usually, the strangeness in hydrodynamical simulations is assumed to be homogeneously distributed, so the condition $n_{S}(x)=\left(n_{S}\right)_{\mathrm{FO}}(x)=0$ binds the parameters associated with strangeness conservation. Equation (3) leaves room to include dynamical strangeness evolution which leads to delocalization of the net strangeness. The possibility of such effects is well established in the literature, e.g., the works on strangeness distillation beginning with works in the 1980s [16] and the measured rapidity distributions of kaons, antikaons, hyperons, and antihyperons, which show at SPS large local differences [17]. In a dynamical model one has to introduce additional dynamical equations to govern the net strangeness flow to account for these processes.

In this limited study, our aim is to describe strange hadron abundances due to the strict treatment of freeze-out conditions. This is an effect that alone influences the post-FO hadron spectrum. Thus, in this work we do not intend to mix up these two effects [see Eq. (8)], although in a full realistic reaction model the strangeness dynamics should also be accounted for.

The process described is generally nonadiabatic, but the entropy constraint

$$
S^{\nu} d \sigma_{\nu} \leqslant S_{\mathrm{FO}}^{\nu} d \sigma_{\nu}
$$

must be satisfied in every point on the FO hypersurface.

\section{A. The statistical post-freeze-out hadronization}

In addition to the surprising success even in the analysis of elementary reactions, one of the important results of the analysis of hadron yield systematics at high energies (SPS and higher) done within the statistical models is the approximate independence of the temperature parameter $(T$ $=160-180 \mathrm{MeV}$ ) of the system size and collision energy. Another important feature found in statistical analyses is the common freeze-out condition, energy per hadron $E / N$ $\sim 1 \mathrm{GeV}$, found for all systems from GSI $2 \mathrm{GeV} A \mathrm{Ni}-\mathrm{Ni}$ to SPS $160 \mathrm{GeV} A \mathrm{~Pb}-\mathrm{Pb}$ collisions [18,1]. These results can be attributed to the statistical character of the hadronization process.

Statistical (thermal) and dynamical (coalescence) type models describe similarly well the final hadron abundances. This "puzzle" was discussed by one of us recently [19], with the conclusion that the similarity of the two results is due to the fact that both approaches are dominated by statistical (degeneracy) factors. Thus, with a few exceptions, hadron abundances can be approximately predicted in the statistical model also, although the time span of the reaction is not sufficient to reach real thermal equilibrium.

Calculations within statistical models are straightforward when the mean number of particles of interest is large, and consequently, it is enough to fulfill the conservation laws in the average sense, i.e., the grand canonical (GC) description can be used. In the GC ensemble, the mean particle multiplicities are just proportional to the volume $V$ of the system. Thus, the particle densities and the ratios of the multiplicities of two different particle species, which are usually used for the comparison with the data, are volume independent.

This simple volume dependence is, however, not valid any more for a small system in which the mean particle multiplicity is low, such as in $p-p$ collisions. In this case the material conservation laws should be imposed exactly on each charge configuration of the system, i.e., canonical ensemble (CE) description should be used. This condition introduces a significant correlation between particles who carry conserved charges (see Refs. [20,21], and references therein).

In $\mathrm{Pb}-\mathrm{Pb}$ collisions at the SPS collider and $\mathrm{Au}-\mathrm{Au}$ reactions at RHIC, we produce large and hot systems, where the corrections are negligible ${ }^{1}$ and GC description is satisfactory for most of the produced particles. Nevertheless, the number of strange particles created is rather low, and the net strangeness is exactly zero. Therefore the CE description would be preferable for the strange particles (we are not going to include particles with charm or heavier quarks). However, the basic assumption of the hydrodynamics is the local thermal and chemical equilibrium, whereas the canonical conservation laws can only be realized globally [because locally, cellby-cell, the conservation laws of the CE (requiring integer numbers of baryon charge and strangeness charge) cannot be realized in a real calculation]. This prevents us using the $\mathrm{CE}$

\footnotetext{
${ }^{1}$ In heavy ion collisions, most of this large volume consists of causally disconnected regions. In cosmology, one speaks in this context of the horizon paradox and introduces "inflation" in order to explain homogeneity of the horizon. Without inflation, the size of causally connected regions is so small that local conservation laws have, in principle, to be considered.
} 
in post-FO, which is nothing but the extension of the hydrodynamical part with different EoS. In kinetic theory this problem has been solved, so conservation laws in the CE can be implemented [22]. This drawback is not of a serious concern, because the conditions reached in high energy heavy ion systems are expected to fulfill the requirements for GC description [21].

In the relativistic $\mathrm{GC}$ ensemble for ideal hadron gas (HG), the properties of matter in unit volume are parametrized by temperature $T$ and fugacities $\lambda_{B}$ and $\lambda_{S}$ (or corresponding chemical potentials) for conserved charges (usually we omit $\lambda_{Q}$ associated with electric charge).

In case of sudden freeze-out, there might not be sufficient time to achieve chemical equilibration of number of strange and antistrange quarks. Thus, an overpopulation or underpopulation of strange hadron species may persist after hadronization. This can, however, be treated in the GC approach as we will see.

Now that the thermal ideal gas of hadrons provides us with tools to calculate post-FO quantities, we can collect the set of equations needed to describe the simultaneous FO and hadronization taking place at a timelike hypersurface. For the sake of simplicity, we choose a hypersurface of constant coordinate time, $d \sigma_{\nu}=(1,0,0,0)$, which is, of course, connected with any timelike hypersurface by a proper Lorentz transformation. Recalling the energy-momentum, baryon current, and strangeness conservation equations yields

$$
\begin{gathered}
(e+P) u_{0}^{2}-P=\left(e_{\mathrm{FO}}+P_{\mathrm{FO}}\right)\left(u_{0}\right)_{\mathrm{FO}}^{2}-P_{\mathrm{FO}}, \\
(e+P) v u_{0}^{2}=\left(e_{\mathrm{FO}}+P_{\mathrm{FO}}\right) v_{\mathrm{FO}}\left(u_{0}\right)_{\mathrm{FO}}^{2}, \\
n_{B} u_{0}=\left(n_{B}\right)_{\mathrm{FO}}\left(u_{0}\right)_{\mathrm{FO}} \\
n_{S} u_{0}=\left(n_{S}\right)_{\mathrm{FO}}\left(u_{0}\right)_{\mathrm{FO}}=0 .
\end{gathered}
$$

Given the pre-FO quantities, the solution of this set of equations gives the post-FO parameters: $v_{\mathrm{FO}}, T_{\mathrm{FO}},\left(\lambda_{B}\right)_{\mathrm{FO}}$, and $\left(\lambda_{S}\right)_{\mathrm{FO}}$, flow velocity of the fluid element, and temperature and fugacities for conserved currents, respectively. These parameters describe completely the hadron spectra and other statistical quantities at the LRF of the fluid element.

In order to take into account the possible overpopulation or underpopulation of strange and antistrange quarks in post-FO side, a parametrization of the conservation of the number of $s, \bar{s}$ pairs must be introduced. In the GC formulation, a conservation law gives rise to a fugacity parameter. The one associated with the number of $s, \bar{s}$ pairs, $n_{s, \bar{s}}$, is usually called $\gamma_{S}$. In Boltzmann approximation, $\gamma_{S}$ is the ratio of actual and equilibrium values of the number of $s, \bar{s}$ pairs [21]. In the original definition [23], $\gamma$ is the ratio of strange and light quark fugacities, $\lambda_{s} / \lambda_{q}$. The relation between these definitions is written as

$$
\gamma_{S}=\frac{n_{s, \bar{s}}}{n_{s, \bar{s}}^{\mathrm{eq}}}=\frac{\lambda_{s}}{\lambda_{s}^{\mathrm{eq}}}=\gamma \frac{\lambda_{q}}{\lambda_{s}^{\mathrm{eq}}},
$$

where the "eq" refers to the complete equilibrium values. The assumption of the survival of $n_{s, \bar{s}}$ over the FO hypersurface yields yet another equation to be included in the set (5)-(8) above:

$$
n_{s, \bar{s}} u_{0}=\left(n_{s, \bar{s}}\right)_{\mathrm{FO}}\left(u_{0}\right)_{\mathrm{FO}} .
$$

Thus, due to Eqs. (8) and (10), the numbers of strange and antistrange quarks are conserved separately during the FO process, and inhomogeneous $S$ distribution is allowed by releasing the local neutrality condition (8).

\section{NUMERICAL STUDIES}

For quantitative studies of the FO, we have chosen a framework of Bjorken model [25] for the expanding QGP. This allows us to cover many FO scenarios with relative ease. Within the Bjorken model, the evolution of matter in one spatial dimension $z$ and charge densities $n_{i}$ is governed by equations

$$
\begin{gathered}
\frac{\partial e}{\partial \tau}+\frac{e+P}{\tau}=0, \\
\frac{\partial P}{\partial y}=0, \\
\frac{\partial n_{i}}{\partial \tau}+\frac{n_{i}}{\tau}=0,
\end{gathered}
$$

where $\tau=\sqrt{t^{2}-z^{2}}$ is the proper time and $y$ is the rapidity of a given fluid element. For vanishing net charges, thermodynamical quantities are constant along constant proper time curves on $(t, z)$ plane. Taking the FO to take place at constant coordinate time, the proper time of freezing out fluid element decreases with increasing spatial coordinate, so the temperature is an increasing function of $z$. The constant coordinate time choice is made in order to have a clear picture of the FO process in different thermal circumstances. In addition to the equations above, one needs an equation to describe the thermodynamical structure of the evolving matter, the equation of state.

\section{A. The equation of state-supercooled QGP}

Two different equations of state for quark-gluon plasma are considered. The QGP is assumed to be an ideal gas of three flavors $(u, d, s)$ of quarks and their antiquarks. We will model the thermodynamics of the QCD vacuum in terms of the MIT bag model [26] by introducing a phenomenological bag constant $B$.

Light quarks $(u, \bar{u}, d, \bar{d})$ can be considered as massless, but the mass of strange quark cannot be neglected. For massless quarks and gluons, integral in partition function $Z_{\mathrm{QGP}}$ can be evaluated analytically, but the contribution of strangeness must be calculated numerically. It is convenient to split $Z_{\mathrm{QGP}}$ into four parts:

$$
\ln Z_{\mathrm{QGP}}=\ln Z_{f}+\ln Z_{b}+\ln Z_{s}+\ln Z_{v a c},
$$


where $\ln Z_{f}, \ln Z_{b}$, and $\ln Z_{s}$ are the contributions of massless quarks, gluons, and massive strange quarks, respectively. $\ln Z_{v a c}$ is the contribution of the bag constant added to the energy-momentum tensor. Evaluation of analytical parts of $\ln Z_{\mathrm{QGP}}$ leads [27] to the expressions

$$
\begin{gathered}
\ln Z_{f}=V\left(\frac{7}{30} \pi^{2} T^{3}+\mu_{q}^{2} T+\frac{1}{2 \pi^{2}} \mu_{q}^{4} \frac{1}{T}\right), \\
\ln Z_{b}=\frac{8}{45} \pi^{2} V T^{3} \\
\ln Z_{v a c}=-\frac{B V}{T}
\end{gathered}
$$

where $\mu_{q}$ is a light quark chemical potential, $\mu_{q}=\mu_{B} / 3$. The total strangeness is zero during the whole collision evolution. If one assumes that the strangeness produced in a collision is spread homogeneously in QGP, then $n_{S}$ must be zero in any given fluid element, so the contribution of $s$ and $\bar{s}$ quarks is

$$
\ln Z_{s}=\frac{6 V}{\pi^{2}} \int_{0}^{\infty} d p p^{2} \ln \left(1+e^{-\beta \sqrt{p^{2}+m_{s}^{2}}}\right)
$$

where $m_{s}$ is the mass of the strange quark, here chosen to vary between 150 and $250 \mathrm{MeV}$.

If one puts the bag constant to zero $(B \equiv 0)$, then $\ln Z_{v a c}$ term vanishes and we end up with the normal quantum distribution for the ideal gas. In this case, the explicit form ${ }^{2}$ of the three-flavor QGP EoS is

$$
P\left(T, \mu_{i}\right)=\frac{1}{3} e, \quad e\left(T, \mu_{i}\right)=e_{S B} .
$$

In the case of finite bag constant, EoS reads

$$
P\left(T, \mu_{B}, B\right)=\frac{1}{3} e_{S B}-B, \quad e\left(T, \mu_{B}\right)=e_{S B}+B .
$$

The third equation of state in addition to ones with finiteand zero-MIT bag constants considered here was suggested in Refs. [28,29] (and first time applied for heavy ion collisions in Ref. [30]) for the case of baryon-free QGP $\left(\mu_{q}\right.$ $=0)$ with two quark flavors:

$$
P(T)=\frac{1}{3} e(T)-b T, \quad e(T)=e_{S B} .
$$

We call this a spinodal EoS, inspired by the existence of a local minimum in pressure profile (20). It is easy to check that $e$ and $P$ are connected by the thermodynamical equation

$$
e=T \frac{d P}{d T}-P .
$$

\footnotetext{
${ }^{2}$ The massive $s, \bar{s}$ quarks give a little correction to this simple rule, but we suppress that in the text.
}

This EoS can be justified as resulting from the nonperturbative QCD effects: The lattice calculations (see, for example, Ref. [31]) show that close to the critical temperature for hadronic matter, QGP phase transition energy density has a sharp increase and soon saturates with an equilibrium StefanBoltzmann value $e(T)=e_{S B}(T)$, while pressure increases much more slowly and reaches Stefan-Boltzmann limit only at very high $T$. There are many recent works on QGP EoS close to deconfinement point with different physics behind: quasiparticle description (for example, Ref. [32]), condensed Polyakov loops (for example, Ref. [33]), and quark-gluon liquid model (for example, Ref. [34]). These models, valid in the region $T>T_{c}$, can quantitatively fit the lattice results mentioned above. In order to illustrate the hadronization processes, we considered several simple model equations of state. The advantage of the spinodal EoS is a simple analytical form, and a possibility to "extrapolate," or just simply use it in the same form in the region $T<T_{c}$. The special feature of this EoS is a local minimum in the pressure profile at some temperature $T=T_{m}\left(T_{m}<T_{c}\right)$.

The spinodal EoS considered here will be of form (20) but with three quark flavors and finite net baryon density:

$$
P\left(T, \mu_{i}\right)=\frac{1}{3} e\left(T, \mu_{i}\right)-b T, \quad e\left(T, \mu_{i}\right)=e_{S B} .
$$

Comparing these expressions with the bag model EoS, one can see that for the pressure the bag constant $B$ is replaced by $T$ - and $\mu$-dependent term $b T$, while for energy density the analogy with bag model disappears due to relation (21).

If the phase transition temperature $T_{c}$ is known, parameter $b$ can be fixed from the Gibbs condition $P_{\mathrm{HG}}\left(T_{c}, \mu\right)$ $=P_{\mathrm{QGP}}\left(T_{c}, \mu\right)$. This same condition fixes the constant $B$ within the bag model.

\section{B. Freeze-out illustrated}

The post-FO matter is described by the quantum ideal gas, composed of hadrons up to a mass of $2.5 \mathrm{GeV}$, listed in the year 2000 issue of Review of Particle Physics [35]. In order to satisfy the conservation equation (10) for strange quark

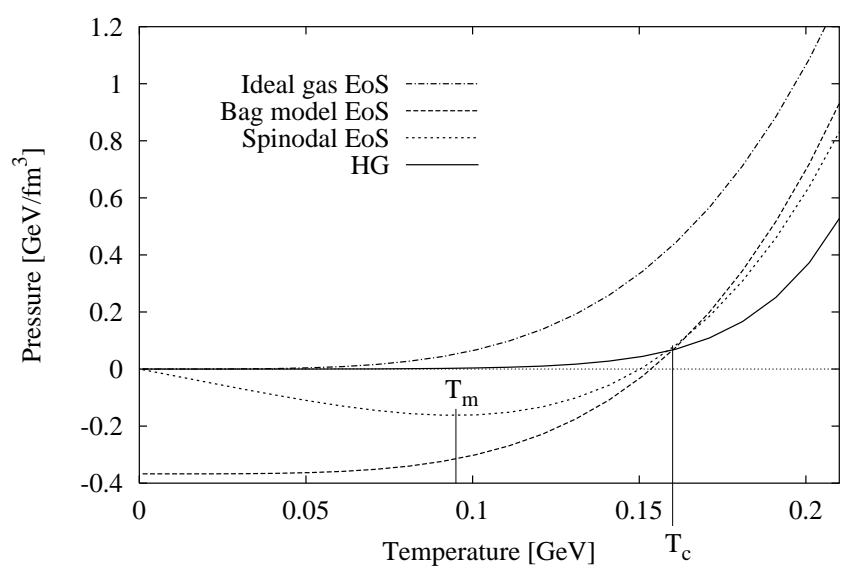

FIG. 1. Hadron gas pressure and quark-gluon plasma pressures with ideal gas, bag model, and spinodal model equations of state with parameters $\mu_{B}=100 \mathrm{MeV}$ and $T_{c}=160 \mathrm{MeV}$. 


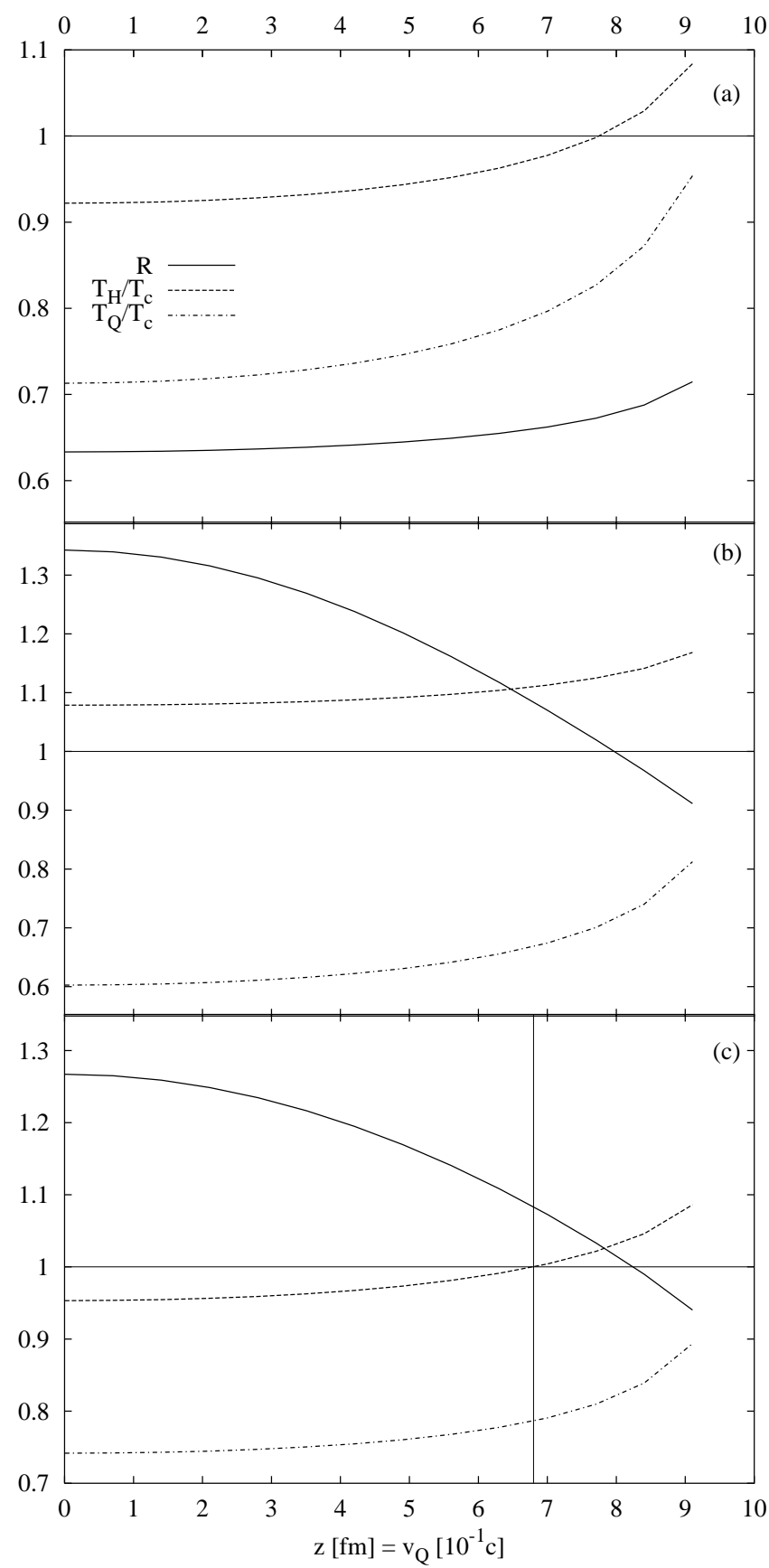

FIG. 2. Various quantities on the FO surface. Panels from top to bottom: (a) ideal gas EoS for QGP, (b) MIT bag EoS, and (c) spinodal EoS. $R$ is the ratio of entropy currents in $\mathrm{HG}$ and QGP, $T_{H}$ and $T_{Q}$ are the temperatures on the HG and QGP side, and $T_{c}$ is the critical temperature. The model parameters in the bag and spinodal EoS are fixed to produce critical temperature at $160 \mathrm{MeV}$. For the ideal gas EoS, there is no real $T_{c}$, the chosen $160 \mathrm{MeV}$ is arbitrary. The vertical line in panel (c) marks the point where the HG temperature crosses the critical value.

pairs, additional fugacity $\gamma_{S}$ is introduced for each strange particle $i$ as $\lambda_{i} \rightarrow \lambda_{i} \gamma_{S}^{\left|S_{i}\right|}$. Additionally, the mesons carrying $s \bar{s}$ pairs must be taken into account. In this work, the number of mesons $(i)$ is affected by the fugacity factor $\lambda_{i}=\gamma_{S}^{2 c_{s}}$, where $c_{s}$ is the relative $s \bar{s}$ content in the meson. We take

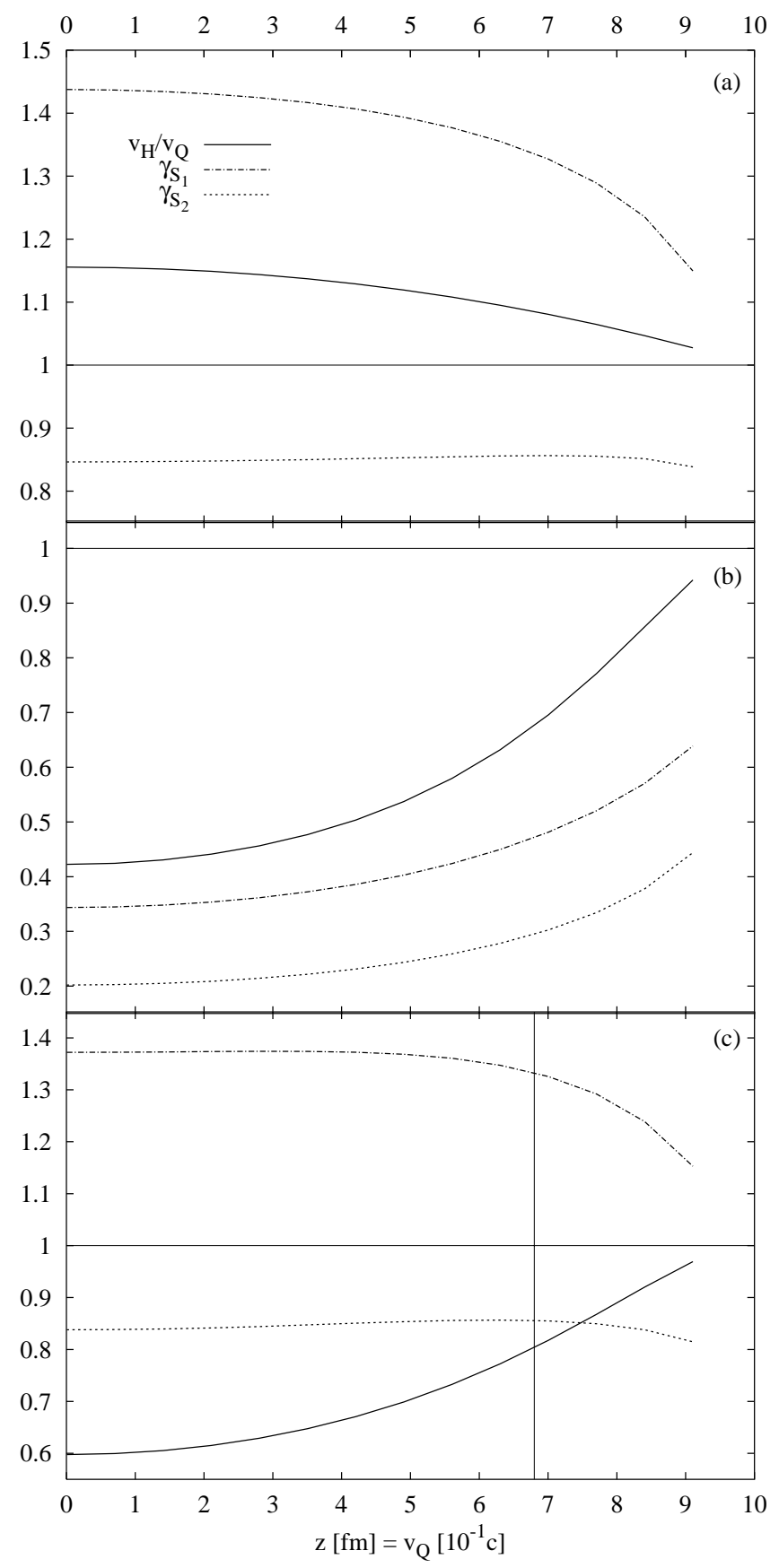

FIG. 3. Various quantities on the FO surface. Panels from top to bottom: (a) ideal gas EoS for QGP, (b) MIT bag EoS, and (c) spinodal EoS. $v_{H}$ and $v_{Q}$ are the HG and QGP flow velocities. $\gamma_{S_{1}}$ and $\gamma_{S_{2}}$ are the strangeness saturation fugacities with $s$ quark masses $150 \mathrm{MeV}$ and $250 \mathrm{MeV}$, respectively. The model parameters in the bag and spinodal EoS are fixed to produce critical temperature at $160 \mathrm{MeV}$. For the ideal gas EoS, there is no real $T_{c}$, the chosen $160 \mathrm{MeV}$ is arbitrary. The vertical line in panel (c) marks the point where the HG temperature crosses the critical value.

$c_{s}=0.5$ for $\eta$ mesons, and $c_{s}=1$ for $\phi, f_{0}(980), f_{1}(1510)$, $\phi(1680)$, and $\phi_{3}(1850)$.

In Fig. 1, we show the pressures of the HG and QGP as functions of temperature. The parameters appearing in different equations of state for QGP are fixed by setting the critical 
temperature to $T_{c}=160 \mathrm{MeV}$. The pressure minimum in the spinodal EoS is labeled by $T_{m}$. Of course, the critical temperature varies with baryon density (or $\mu_{B}$ ), but we find this variation to be negligible within reasonable range of FO density. The strangeness saturation parameter $\gamma_{S}$ is set to 1 , corresponding to the full strangeness equilibration. We always let the QGP side be in full strangeness equilibrium, so the Gibbs condition for the adiabatic, isothermic phase transition compels $\gamma_{S}=1$. In the following, however, the phase transition is neither isothermic nor adiabatic, so the change in fugacities is allowed and unavoidable.

Figure 2 depicts the variation of some relevant variables along the coordinate time $t=10 \mathrm{fm} / c$ in the Bjorken model. Going from the edge of the light cone $(z=10 \mathrm{fm})$ to the nonflowing center of the system $(z=0 \mathrm{fm})$, we describe the changes of quantities in proper time of the given fluid element from $\tau=0$ to $\tau=10$. That is, going from the origin to the right, we go from the later, cooler and more dilute stage of evolution to the earlier, hotter and more dense stage. We have chosen the initial values for energy density and baryon density as $e_{0}=5 \mathrm{GeV} \mathrm{fm}^{-3}$ and $\left(n_{B}\right)_{0}=1 \mathrm{fm}^{-3}$, respectively, at the time $\tau=1 \mathrm{fm} / c$. For the ideal gas QGP EoS, the higher $e_{0}=8 \mathrm{GeV}$ is used in order to keep the figure more descriptive. The model parameters $B$ in the MIT bag EoS and $b$ in the spinodal EoS are fixed to produce critical temperature at $160 \mathrm{MeV}$, while for the ideal gas EoS there is no critical temperature. The arbitrary value $T_{c}=160 \mathrm{MeV}$ is chosen to give similar scaling for temperatures in all cases. For realistic FO conditions, the ratio $R$ of entropy currents in HG and QGP is well above unity for bag and spinodal EoS. A more realistic form of the QGP equation of state may alter this behavior. Or, considering that there is insufficient time for substantial entropy production to occur in a sudden hadronization process, the hadrons could be produced far from chemical equilibrium [23,24]. For the reference, the $R$ for the ideal gas QGP is, as expected, far below one. The crossing of HG temperature $T_{H}$ with $T_{c}$ marks the end point of the physically allowed FO. For the bag model, there is no such crossing, but the FO stays unphysical with all reasonable values of QGP temperature $T_{Q}$. We could choose lower initial value for the QGP energy density, but the degree of supercooling, $T_{Q} / T_{c}$, would be of order 0.5 at the $\mathrm{HG}$ critical point. For the spinodal model QGP, the case is different. At the point $T_{H} / T_{c}=1$, the degree of QGP supercooling is about $24 \%$, and stays rather constant when going towards the cooler system.

In Fig. 3 we illustrate the changes in flow velocities and strangeness saturation parameter in the circumstances equal to the ones in Fig. 2. For both cases, bag and spinodal QGP, the flow is decelerated at the FO to $\mathrm{HG}$, indicated by the ratio of $\mathrm{HG}$ and QGP velocities, $v_{H} / v_{Q}$. At the point $T_{H}$ $=160 \mathrm{MeV}$ for the spinodal EoS, this ratio is 0.80 , indicating the final HG flow velocity of $0.54 c$. The $\gamma_{S}$, resulting from the survival of $s \bar{s}$ pairs through the FO process, is calculated for two different values of $s$ quark mass $m_{s} \cdot \gamma_{S_{1}}$ corresponds to $m_{s}=150 \mathrm{MeV}$ and $\gamma_{S_{2}}$ is for $m_{s}=250 \mathrm{MeV}$. Although $\gamma_{S}$ is always 1 on the quark side, for small $m_{s}$ we have a larger $s, \bar{s}$ number that for the larger strange quark mass that is well above the freeze-out temperature. This strange quark pair number remains unchanged during the freeze-out, and so for the smaller mass we have an oversaturation of strangeness on the hadronic side, $\gamma_{S 1} \sim 1.4$ [for EoS, Fig. 3(c)]. In case of the large strange quark mass we have less strange quarks, which leads to strangeness undersaturation on the hadronic side compared to the hadronic equilibrium, $\gamma_{S 2} \sim 0.85$ (for the same EoS). Comparing the figures, we find $\gamma_{S}$ to be very sensitive to the choice of QGP EoS and the $m_{s}$. It is worth noting that fixing $\gamma_{S}$ to one or varying the $m_{s}$ gives only negligible change to other quantities in Figs. 2 and 3.

\section{CONCLUSIONS}

We have found that the realistic and accurate study of the freeze-out process in hydrodynamical models is important and cannot be neglected. Our results show that the FO process is very sensitive on the properties of the EoS. Since our main goal is to identify the EoS from the data, this is an observation of basic importance.

The correct treatment shows that FO is not even possible from an arbitrary kind of initial state, and the entropy constraint is a sensitive condition.

From the point of strangeness, we can also conclude that strangeness production is very sensitive to the correct FO treatment and to the pre-FO EoS. Thus, strangeness data not only provide a signal of QGP formation, but with proper and realistic description of freeze-out, strangeness provides the most sensitive signal indicating different properties of the pre-FO EoS.

In conclusion, this study shows that collective, continuum reaction models, such as fluid dynamical (FD) models (onefluid FD, multifluid FD, and chiral FD), can and must be supplemented with realistic freeze-out treatment to evaluate measurable data. These calculations indicate that this is now possible cell by cell in FD models, although it requires more than average computational capacity and needs preferably high performance parallel computing.

\section{ACKNOWLEDGMENTS}

We acknowledge the constructive discussions with Esko Suhonen. Two of us (A.K. and V.M.) acknowledge the support of the Bergen Computational Physics Laboratory in the framework of the European Community-Access to Research Infrastructure action of the Improving Human Potential Program. 
[1] F. Becattini, J. Cleymans, A. Keränen, E. Suhonen, and K. Redlich, Phys. Rev. C 64, 024901 (2001).

[2] J. Cleymans, D. Elliott, R.L. Thews, and H. Satz, Z. Phys. C 74, 319 (1997).

[3] P. Braun-Munzinger, I. Heppe, and J. Stachel, Phys. Lett. B 465, 15 (1999).

[4] J. Cleymans and K. Redlich, Phys. Rev. Lett. 81, 5284 (1998).

[5] J. Cleymans, D. Elliott, A. Keränen, and E. Suhonen, Phys. Rev. C 57, 3319 (1998).

[6] J. Cleymans, A. Keränen, M. Marais, and E. Suhonen, Phys. Rev. C 56, 2747 (1997).

[7] F. Becattini, Z. Phys. C 69, 485 (1996).

[8] F. Becattini and U. Heinz, Z. Phys. C 76, 269 (1997).

[9] L.P. Csernai and J.I. Kapusta, Phys. Rev. D 46, 1379 (1992).

[10] L.P. Csernai and J.I. Kapusta, Phys. Rev. Lett. 69, 737 (1992).

[11] T. Csörgó and L.P. Csernai, Phys. Lett. B 333, 494 (1994).

[12] Cs. Anderlik et al., Phys. Rev. C 59, 388 (1999); 59, 3309 (1999); Phys. Lett. B 459, 33 (1999).

[13] V.K. Magas et al., Heavy Ion Phys. 9, 193 (1999); Nucl. Phys. A661, 596 (1999).

[14] J. Rafelski and J. Letessier, Phys. Rev. Lett. 85, 4695 (2000).

[15] J. Rafelski and B. Müller, Phys. Rev. Lett. 48, 1066 (1982).

[16] C. Greiner, P. Koch, and H. Stöcker, Phys. Rev. Lett. 58, 1825 (1987); C. Greiner, D. Rischke, P. Koch, and H. Stöcker, Phys. Rev. D 38, 2797 (1988).

[17] J. Rafelski and J. Letessier, Nucl. Phys. A702, 304 (2002).

[18] J. Cleymans and K. Redlich, Phys. Rev. Lett. 81, 5284 (1998).

[19] L.P. Csernai, J. Phys. G 28, 1993 (2002).

[20] J. Cleymans, A. Keranen, and E. Suhonen, Proceedings of the 11th Chris Engelbrecht Summer School in Theoretical Physics, Cape Town, 1998 (Springer-Verlag, Berlin, 1999).

[21] A. Keränen and F. Becattini, Phys. Rev. C 65, 044901 (2002).

[22] L. Turko and J. Rafelski, Eur. Phys. J. C 18, 587 (2001); C.M. Ko et al., Phys. Rev. Lett. 86, 5438 (2001).

[23] P. Koch, B. Müller, and J. Rafelski, Phys. Rep. 142, 167 (1986).

[24] J. Rafelski and J. Letessier, hep-ph/0206145.

[25] J.D. Bjorken, Phys. Rev. D 27, 140 (1983).

[26] A. Chodos, R.L. Jaffe, K. Johnson, and C.B. Thorn, Phys. Rev. D 10, 2599 (1974).

[27] J. Cleymans, R.V. Gavai, and E. Suhonen, Phys. Rep. 130, 217 (1986).

[28] C.G. Kallman, Phys. Lett. 134B, 363 (1984).

[29] M.I. Gorenstein and O.A. Mogilevsky, Z. Phys. C 38, 161 (1988).

[30] L.L. Jenkovszky, B. Kampfer, and V.M. Sysoev, Z. Phys. C 48, 147 (1990).

[31] T. Blum, L. Kärkkäinen, and D. Toussaint, Phys. Rev. D 51, 5153 (1995).

[32] A. Peshier, B. Kämpfer, and G. Soff, Phys. Rev. C 61, 045203 (2000).

[33] O. Scavenius, A. Dumitru, and J.T. Lenaghan, hep-ph/0201079.

[34] S. Hamieh, J. Letessier, and J. Rafelski, Phys. Rev. C 62, 064901 (2000).

[35] Particle Data Group, D.E. Groom et al., Eur. Phys. J. C 15, 1 (2000). 